\title{
ITERATION OF A COMPOSITION OF EXPONENTIAL FUNCTIONS
}

\author{
XIAOYING DONG
}

AbStract. We show that for certain complex parameters $\lambda_{1}, \ldots, \lambda_{n-1}$ and $\lambda_{n}$ the Julia set of the function

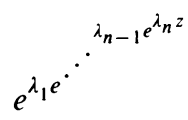

is the whole plane $\mathbb{C}$. We denote by $\Lambda$ the set of $n$-tuples $\left(\lambda_{1}, \ldots, \lambda_{n}\right)$, $\lambda_{1}, \ldots, \lambda_{n} \in \mathbb{R}$ for which the equation<smiles>O=C=CC=CC(=O)O</smiles>

has exact two real solutions. In fact, one of them is an attracting fixed point of

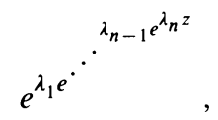

which is denoted by $q$. We also show that when $\left(\lambda_{1}, \ldots, \lambda_{n}\right) \in \Lambda$, the Julia set of

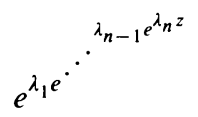

is the complement of the basin of attraction of $q$. The ideas used in this note may also be applicable to more general functions.

\section{A continued composition of $n$ exponential functions}

$$
f_{i}(z)=e^{\lambda_{i} z}, \quad \lambda_{i} \in \mathbb{C}, i=1,2, \ldots, n,
$$

shall be denoted by the symbol $E_{\lambda_{1}, \ldots, \lambda_{n}}$ which is an abbreviation for

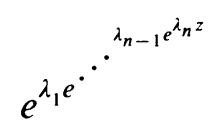

that is, each $e^{\lambda_{i}}$ is used as the exponent of the preceding.

It had until 1981 been an open problem, proposed by Fatou [9], whether $\mathscr{T}\left(e^{z}\right)=\mathbb{C}$. In 1981 Misiurewicz [11] proved this conjecture answering this sixty year old question of Fatou. In 1984, Baker and Rippon [4] studied the

Received by the editors July 15, 1989 .

1980 Mathematics Subject Classification (1985 Revision). Primary 30D05; Secondary 58F08. 
sequence of iterations of $E_{a}(z)=e^{a z}$ where $a$ is a nonzero complex parameter (similar results were obtained by Devaney [5]). They analysed the way in which $\mathscr{N}\left(E_{a}\right)$ divides the plane and the various possible limit functions for convergent subsequences of $\left\{E_{a}^{n}\right\}_{n=0}^{\infty}$ in components of $\mathscr{N}\left(E_{a}\right)$. They proved that there are no limit functions (and so $\mathscr{T}\left(E_{a}\right)=\mathbb{C}$ ) provided $\lim _{n \rightarrow \infty} E_{a}^{n}(0)=\infty$, in particular this is the case for all real $a>\frac{1}{e}$. This extends the result of Misiurewicz for $a=1$. In this paper we study the Julia set of the function

$$
E_{\lambda_{1}, \ldots, \lambda_{n}}(z)=e^{\lambda_{1} e \cdot{ }^{\cdot \lambda_{n-1} e^{\lambda_{n} z}}}
$$

where $\lambda_{1}, \ldots, \lambda_{n}$ are complex parameters, and extend Baker and Rippon's result for the case $n=1$. Some aspects of the convergence of the sequence of the natural iterates of this function were also studied by Thron [13] in 1957 and Shell [12] in 1959.

The Julia set of an entire function $f$ can be defined as either the set of points at which the family $\left\{f^{n}\right\}_{n=0}^{\infty}$ of iterations of $f$ is not normal or as the closure of the set of repelling periodic points [1].

Throughout this paper we denote the set of complex numbers by $\mathbb{C}$, the set of real numbers by $\mathbb{R}$, the Julia set of $E_{\lambda_{1}, \ldots, \lambda_{n}}$ by $\mathscr{T}(E)$ and the normal set of $E_{\lambda_{1}, \ldots, \lambda_{n}}$ by $\mathscr{N}(E)$.

We need the following known results:

Theorem A. Unless $f(z)$ is a rational function of order 0 or 1 the set $\mathscr{T}(f)$ has the following properties (proved for rational functions in $[7,8]$ and for entire functions in [9]):

(1) $\mathscr{T}(f)$ is a nonempty perfect set.

(2) $\mathscr{T}\left(f^{n}\right)=\mathscr{T}(f)$ for any integer $n \geq 1$.

(3) $\mathscr{T}(f)$ is completely invariant under the mapping $z \rightarrow f(z)$, i.e., if $\alpha$ belongs to $\mathscr{T}(f)$ then so do $f(\alpha)$ and every solution $\beta$ of $f(\beta)=\alpha$.

Theorem B. Let $D$ be a domain of the complex plane with at least three boundary points and let $f$ be analytic in $\bar{D}$, except that if $D$ is unbounded $f$ need not be analytic at $\infty$. Let $f$ map $D$ into itself and suppose that no subsequence of $\left\{f^{n}\right\}_{n=0}^{\infty}$ has the identity map as a limit in $D$ (in particular this is so if $f$ is not a univalent map of $D$ onto $D)$. Then the whole sequence $\left\{f^{n}\right\}_{n=0}^{\infty}$ converges in $D$ to a constant limit $\alpha \in \bar{D}$ [3].

We denote by $\mathscr{S}$ the set of all finite singularities of $f^{-1}(z)$ and $\mathscr{E}$ the set of points of the form $f^{n}(s), s \in \mathscr{S}, n=0,1,2, \ldots$. Then a point belongs to $\mathscr{E}$ precisely when it is a finite singularity of some inverse function $f^{-n}(z)$ of an iterate of $f(z)$ [2].

To study the stable behavior of a transcendental entire function $f$ we need to discuss the possible limit functions of subsequences of $\left\{f^{n}\right\}_{n=0}^{\infty}$ in the domains concerned. The following results are developed by Baker [2]. 
Theorem C. Let $\mathscr{E}^{\prime}$ denote the derived set of $\mathscr{E}$, then any constant limit of a sequence $\left\{f^{n_{k}}(z)\right\}_{k=0}^{\infty}$ in a component of the normal set $\mathscr{N}(f)$ belongs to

$$
\mathscr{L}=\mathscr{E} \cup \mathscr{E}^{\prime} \cup\{\infty\}=\overline{\mathscr{E}} \cup\{\infty\}
$$

Theorem D. If the set $\mathscr{L}$ defined in Theorem $\mathrm{C}$ has an empty interior and a connected complement, then no sequence $\left\{f^{n_{k}}\right\}_{k=0}^{\infty}$ has a nonconstant limit function in any component of $\mathcal{N}(f)$.

A point $\omega \in \widehat{\mathbb{C}}$ is an asymptotic value for a map $f$ if there is a path $\alpha:[0,1) \rightarrow \mathbb{C}$ such that $\lim _{t \rightarrow 1} \alpha(t)=\infty$ and $\lim _{t \rightarrow 1} f \circ \alpha(t)=\omega$.

Let $U$ be a connected component (domain) of $\mathscr{N}(f) . U$ is preperiodic if there exist integers $p$ and $q$ such that $f^{p+q}(U)=f^{p}(U)$; it is periodic if $p=0$. A component of $\mathscr{N}(f)$ which is neither periodic nor preperiodic is wandering.

A map $f$ is called of critically finite type (or simply finite type) if $f$ has finitely many critical values and asymptotic values. The following theorem is due to L. Goldberg and L. Keen, and describes the stable behavior of finite type entire functions [10].

Theorem E. If $f: \mathbb{C} \rightarrow \mathbb{C}$ is of finite type, then $f$ has no wandering domain.

We remark that the application of the last result mentioned above is essential in the proof of the following theorem:

Theorem 1. Let $\mathscr{S}$ be the set of the finite singularities of the inverse function of $E_{\lambda_{1}, \ldots, \lambda_{n}}$. If each forward orbit of $s \in \mathscr{S}$ tends to $\infty$, then $\mathscr{T}(E)=\mathbb{C}$.

In particular, if $\lambda_{i}>0$ for $i=1,2, \ldots, n$ and the forward orbit of 0 tends to $\infty$, then $\mathscr{T}(E)=\mathbb{C}$.

In order to prove the theorem we need the following lemmas.

Lemma 2. Finite type maps are closed under composition.

Proof. Assume that two maps $f$ and $g$ are of finite type.

If $\alpha$ is a critical value of $f(g(z))$, then there exists $\beta$ such that

$$
f^{\prime}(g(\beta)) g^{\prime}(\beta)=0 \text { and } f(g(\beta))=\alpha .
$$

If $f^{\prime}(g(\beta))=0$, then $\alpha$ is a critical value of $f$ as well; if $g^{\prime}(\beta)=0$, then there is at least one critical value of $g$ corresponding to $\alpha$ under $f$. It follows that the number of critical values of $f(g(z))$ is less than or equal to the sum of the critical values of $f$ and $g$.

If $\alpha$ is an asymptotic value of $f(g(z))$, then there exists a critical path $\Gamma:[0,1) \rightarrow \mathbb{C}$ such that

$$
\lim _{t \rightarrow 1} \Gamma(t)=\infty \text { and } \quad \lim _{t \rightarrow 1} f(g(\Gamma(t)))=\alpha .
$$

We claim that there exists $\gamma$ which is either a finite number or $\infty$ such that

$$
\lim _{t \rightarrow 1} g(\Gamma(t))=\gamma
$$


Otherwise, let us denote by $M$ the set of the limiting points of $g(\Gamma(t))$ as $t \rightarrow 1$. Then $f(z)=\alpha$ for each $z \in M$. Since $f$ is a nonconstant entire map, $M$ does not contain any limit points, that is $M$ is a discrete set. Suppose that $M$ contains more than one point. Arbitrarily pick two different points $\gamma_{1}, \gamma_{2} \in M$, then they can be separated by two disjoint closed discs $D_{1}$ and $D_{2}$ with $\gamma_{i} \in D_{i}$ where $i=1,2$ and

$$
\left(D_{1} \cup D_{2} \backslash\left\{\gamma_{1}, \gamma_{2}\right\}\right) \cap M=\varnothing \text {. }
$$

But the fact that each $\gamma_{i}$ is a limiting point implies that the curve $g(\Gamma(t))$ where $t \in[0,1)$ must frequently enter each disc $D_{i}$ in fact infinitely many times. Thus the curve $g(\gamma(t))$ where $t \in[0,1)$ intersects the circles $C_{i}$ which are the boundaries of $D_{i}$ infinitely many times as $t \rightarrow 1$. Since $C_{1}$, say, is compact, there must be a limiting point $\gamma_{0}$ on it, and this limit point does not belong to $M$. Contradiction! Therefore $M$ consists of a single point $\gamma$ which is an asymptotic value of $g$. Since $g$ is continuous, the image of $\Gamma$ is also a path. Thus the number of the asymptotic values of $f(g(z))$ is less than or equal to the number of the asymptotic values of $g(z)$. Q.E.D.

As an immediate consequence, $E_{\lambda_{1}, \ldots, \lambda_{n}}$ is of finite type.

Lemma 3. The only finite singularities of $E_{\lambda_{1}, \ldots, \lambda_{n}}^{-1}$ are

$$
0,1, e^{\lambda_{1}}, e^{\lambda_{1} e^{\lambda_{2}}}, \ldots, e^{\lambda_{1} e^{.} \cdot{ }^{\lambda_{n-1} e^{\lambda_{n}}}}
$$

Proof. This follows from the simple facts that $E_{\lambda_{1}, \ldots, \lambda_{n}}$ does not have any algebraic singularity and the inverse function

$$
E_{\lambda_{1}, \ldots, \lambda_{n}}^{-1}(z)=\frac{1}{\lambda_{n}} \ln \left(\frac{1}{\lambda_{n-1}} \ln \left(\cdots\left(\frac{1}{\lambda_{1}} \ln z\right) \cdots\right)\right)
$$

is well defined if and only if $z \neq 0,1, e^{\lambda_{1}}, e^{\lambda_{1} e^{\lambda_{2}}}, \ldots, e^{\lambda_{1} e^{\cdot} \cdot} \quad$ Q.E.D.

Therefore, with Baker's notation

$$
\mathscr{S}=\left\{0,1, e^{\lambda_{1}}, e^{\lambda_{1} e^{\lambda_{2}}}, \ldots, e^{\lambda_{1} e^{\cdot} \cdot{ }^{\lambda_{n-1} e^{\lambda_{n}}}}\right\}
$$

and

$$
\mathscr{L}=\operatorname{Closure}\left\{E_{\lambda_{1}, \ldots, \lambda_{n}}^{k}(s), s \in \mathscr{S}, k=1,2, \ldots\right\} .
$$

We are going to show the following lemma.

Lemma 4. Under the assumptions of Theorem 1, the complement of $\mathscr{L}$ is connected.

Proof. It suffices to show that if $P$ is a countable subset of $\mathbb{C}$, then the complement $\mathbb{C} \backslash P$ of $P$ is connected. Towards this end, pick arbitrarily two points 
$q_{1}, q_{2} \in \mathbb{C} \backslash P$. Through each $q_{i}, i=1,2$, there exists a family $L_{i}$ of uncountably many straight lines. There must be $l_{i} \in L_{i}, i=1,2$, such that $l_{1}$ and $l_{2}$ have an intersection and contain no points of $P$. It follows from the fact that $\mathbb{C} \backslash P$ is path-connected that $\mathbb{C} \backslash P$ is connected. Q.E.D.

Now we are ready to prove Theorem 1:

Proof of the Theorem 1. The set $\mathscr{L}$ has empty interior and connected complement. According to a theorem of I. N. Baker [2], each limit function of the family $\left\{E_{\lambda_{1}, \ldots, \lambda_{n}}^{k}\right\}_{k=0}^{\infty}$ must be either a constant belonging to $\mathscr{L}$ or $\infty$.

It remains to show that the family $\left\{E_{\lambda_{1}, \ldots, \lambda_{n}}^{k}\right\}_{k=0}^{\infty}$ is normal nowhere.

Suppose that $\mathscr{N}(E)$ is not empty. Let $U$ be a component of $\mathscr{N}(E)$. Since $E_{\lambda_{1}, \ldots, \lambda_{n}}$ is critically finite, $E_{\lambda_{1}, \ldots, \lambda_{n}}$ does not possess a wandering domain [6]. Thus there exist nonnegative integers $l$ and $m$ such that $G=E_{\lambda_{1}, \ldots, \lambda_{n}}^{m}(U)$ is invariant under $g=E_{\lambda_{1}, \ldots, \lambda_{n}}^{l}$.

It follows from Theorem B that the whole sequence $\left\{g^{k}\right\}_{k=0}^{\infty}$ converges in $G$ to a constant limit which belongs to $\bar{G}$. Denote this limit by $\alpha$. Since $G$ is invariant under $g$, if $\alpha$ is finite, we have $g(\alpha)=\alpha$. This is to say that $\alpha$ is periodic.

By the hypothesis of the theorem it is clear that for each $s \in \mathscr{S}$,

$$
\lim _{k \rightarrow \infty} E_{\lambda_{1}, \ldots, \lambda_{n}}^{k}(s)=\infty \text {. }
$$

Thus $s \in \mathscr{S}$ is not eventually periodic, and

$$
\begin{aligned}
\mathscr{L} & =\operatorname{Closure}\left\{E_{\lambda_{1}, \ldots, \lambda_{n}}^{k}(s), s \in \mathscr{S}, k=1,2, \ldots\right\} \\
& =\left\{E_{\lambda_{1}, \ldots, \lambda_{n}}^{k}(s), s \in \mathscr{S}, k=1,2, \ldots\right\} \cup\{\infty\} .
\end{aligned}
$$

Applying Theorem C, $\alpha \in \mathscr{L} \backslash\{\infty\}$ cannot be periodic. Contradiction! Therefore, $\alpha$ must be $\infty$.

It follows from

$$
\lim _{k \rightarrow \infty} E_{\lambda_{1}, \ldots, \lambda_{n}}^{k l}(z)=\lim _{k \rightarrow \infty} g^{k}(z)=\infty
$$

uniformly on $G$ that

$$
\lim _{k \rightarrow \infty} E_{\lambda_{1}, \ldots, \lambda_{n}}^{k l-1}(z)=\infty
$$

uniformly on $G$.

Consequently, for each $j \geq 0$,

$$
\lim _{k \rightarrow \infty} E_{\lambda_{1}, \ldots, \lambda_{n}}^{k l-j}(z)=\infty
$$

uniformly on $G$.

For each sequence

$$
\left\{E_{\lambda_{1}, \ldots, \lambda_{n}}^{k_{m}}\right\}_{m=0}^{\infty} \subset\left\{E_{\lambda_{1}, \ldots, \lambda_{n}}^{k}\right\}_{k=0}^{\infty},
$$


there exists an integer $j \geq 0$ such that there is a subsequence

$$
\left\{E_{\lambda_{1}, \ldots, \lambda_{n}}^{k_{m_{t}}}\right\}_{t=0}^{\infty} \subset\left\{E_{\lambda_{1}, \ldots, \lambda_{n}}^{k_{m}}\right\}_{m=0}^{\infty},
$$

which is a subsequence of $\left\{E_{\lambda_{1}, \ldots, \lambda_{n}}^{k l-j}\right\}_{k=0}^{\infty}$. Thus we have

$$
\lim _{t \rightarrow \infty} E_{\lambda_{1}, \ldots, \lambda_{n}}^{k_{m_{t}}}(z)=\infty
$$

in $G$. Furthermore we conclude that the whole sequence $\left\{E_{\lambda_{1}, \ldots, \lambda_{n}}^{k}\right\}_{k=0}^{\infty}$ has $\operatorname{limit} \infty$.

Now we claim that the sequence $\left\{\left(E_{\lambda_{1}, \ldots, \lambda_{n}}^{k}\right)^{\prime}\right\}_{k=0}^{\infty}$ of derivatives of $E_{\lambda_{1}, \ldots, \lambda_{n}}^{k}$, $k=0,1, \ldots$, also tends to $\infty$ on $G$. In fact,

$$
\left(E_{\lambda_{1}, \ldots, \lambda_{n}}\right)^{\prime}(z)=\prod_{i=1}^{n} \lambda_{i} E_{\lambda_{i}, \ldots, \lambda_{n}}(z),
$$

and so, according to the chain rule

$$
\begin{aligned}
\left(E_{\lambda_{1}, \ldots, \lambda_{n}}^{k}\right)^{\prime}(z) & =\prod_{j=1}^{k}\left(E_{\lambda_{1}, \ldots, \lambda_{n}}\right)^{\prime}\left(E_{\lambda_{1}, \ldots, \lambda_{n}}^{j-1}(z)\right) \\
& =\prod_{i=1}^{n} \prod_{j=0}^{k-1} \lambda_{i}^{k} E_{\lambda_{i}, \ldots, \lambda_{n}}\left(E_{\lambda_{1}, \ldots, \lambda_{n}}^{j}(z)\right) .
\end{aligned}
$$

Therefore,

$$
\ln \left|\left(E_{\lambda_{1}, \ldots, \lambda_{n}}^{k}\right)^{\prime}(z)\right|=k \sum_{i=1}^{n} \ln \left|\lambda_{i}\right|+\sum_{j=0}^{k-1} \sum_{i=1}^{n} \ln \left|E_{\lambda_{i}, \ldots, \lambda_{n}}\left(E_{\lambda_{1}, \ldots, \lambda_{n}}^{j}(z)\right)\right| .
$$

Since

$$
\begin{aligned}
\lim _{k \rightarrow \infty}\left|E_{\lambda_{1}, \ldots, \lambda_{n}}^{k+1}(z)\right| & =\lim _{k \rightarrow \infty}\left|E_{\lambda_{1}, \ldots, \lambda_{n}}\left(E_{\lambda_{1}, \ldots, \lambda_{n}}^{k}(z)\right)\right| \\
& =\lim _{k \rightarrow \infty}\left|e^{\lambda_{1} E_{\lambda_{2}, \ldots, \lambda_{n}}\left(E_{\lambda_{1}, \ldots, \lambda_{n}}^{k}(z)\right)}\right|=\infty,
\end{aligned}
$$

for $z \in G$, it follows that

$$
\lim _{k \rightarrow \infty}\left|E_{\lambda_{2}, \ldots, \lambda_{n}}\left(E_{\lambda_{1}, \ldots, \lambda_{n}}^{k}(z)\right)\right|=\infty .
$$

With a similar argument, consequently we have

$$
\lim _{k \rightarrow \infty}\left|E_{\lambda_{i}, \ldots, \lambda_{n}}\left(E_{\lambda_{1}, \ldots, \lambda_{n}}^{k}(z)\right)\right|=\infty
$$

for $i=1, \ldots, n$, which implies that

$$
\lim _{k \rightarrow \infty} \ln \left|\left(E_{\lambda_{1}, \ldots, \lambda_{n}}^{k}\right)^{\prime}(z)\right|=\infty
$$

and our assertion follows immediately. It follows from the Bloch-Landau Theorem that if $D$ is a disc contained in $G$, then $E_{\lambda_{1}, \ldots, \lambda_{n}}^{k}(D)$ contains a disk of 
arbitrarily large radius. Since $\mathscr{T}(E) \neq \varnothing$, there exists an integer $k_{0}>0$ such that

$$
E_{\lambda_{1}, \ldots, \lambda_{n}}^{k_{0}}(D) \cap \mathscr{T}(E) \neq \varnothing
$$

which is impossible, since $D \subset G \subset \mathscr{N}(E)$.

In the case where all $\lambda_{i}>0$, the argument is much simpler since the fact that

$$
\lim _{k \rightarrow \infty} E_{\lambda_{1}, \ldots, \lambda_{n}}^{k}(0)=\infty
$$

implies that each forward orbit of a real number tends to $\infty$. Particularly, each forward orbit of $s \in \mathscr{S} \subset \mathbb{R}$ tends to $\infty$, and the result follows immediately. Q.E.D.

Now we focus our attention on the case when $\lambda_{i}>0, i=1,2, \ldots, n$, and $E_{\lambda_{1}, \ldots, \lambda_{n}}$ has exactly two distinct positive fixed points. From the convexity of the graph of $E_{\lambda_{1}, \ldots, \lambda_{n}}$, for $z$ real it follows that of these one is attracting and the other is repelling.

We denote the attracting one by $q$ and the repelling one by $p$ as shown in Figure 1 (where the dotted line signifies the horizontal asymptote of the function). Also, from the convexity of $E_{\lambda_{1}, \ldots, \lambda_{n}}$, clearly $q>p$.

Noting that there exists $\varepsilon>0$ such that

$$
\left|E_{\lambda_{1}, \ldots, \lambda_{n}}^{\prime}(p)\right|>1+\varepsilon
$$

We have the following theorem:

Theorem 6. Let $\lambda_{i}>0, i=1,2, \ldots, n$. If $E_{\lambda_{1}, \ldots, \lambda_{n}}$ has an attracting fixed point $q$, then $\mathscr{T}(E)$ is the complement of the basin of attraction of $q$.

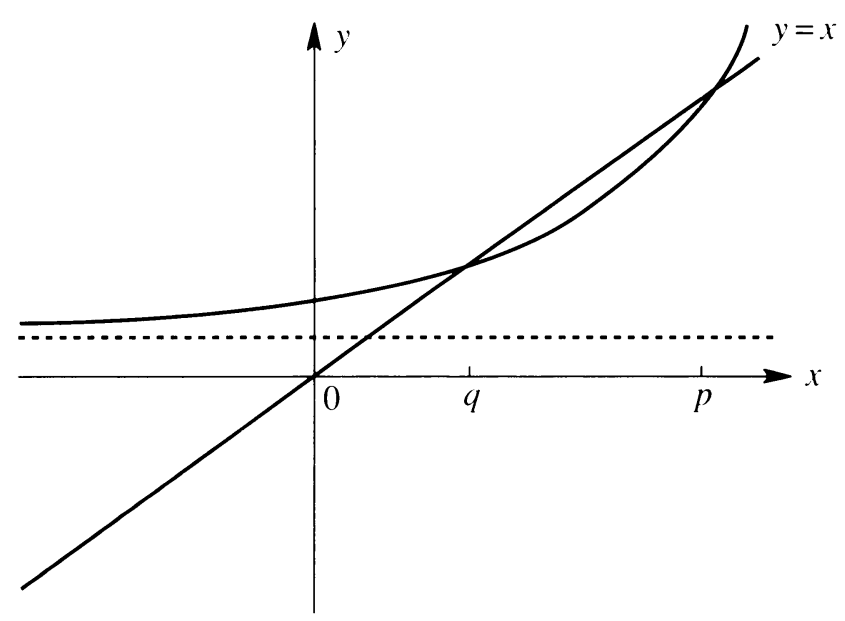

FIGURE 1 
Proof. Write $z=x+i y$ with $x, y \in \mathbb{R}$. Let $H=\{z \in \mathbb{C} \mid x<p\}$. Since, for $z \in H$,

$$
\begin{aligned}
\left|E_{\lambda_{1}, \ldots, \lambda_{n}}(z)\right| & =\left|E_{\lambda_{1}, \ldots, \lambda_{n-1}}\left(e^{\lambda_{n} x+i \lambda_{n} y}\right)\right| \\
& \leq E_{\lambda_{1}, \ldots, \lambda_{n-1}}\left(e^{\lambda_{n} x}\right)<E_{\lambda_{1}, \ldots, \lambda_{n}}(p)=p,
\end{aligned}
$$

the images of $H$ under $\left\{E_{\lambda_{1}, \ldots, \lambda_{n}}^{k}\right\}_{k=0}^{\infty}$ are bounded by the disc centered at 0 with radius $p$. Clearly the interval $[0, q] \subset H$ is contained in the basin of attraction of $q$. It follows from Vitali's Convergence Theorem that $H$ is included in the basin of attraction of $q$. Hence $\mathscr{T}(E)$ lies to the right of the vertical line $x=p .^{1}$ Furthermore we can show that $\mathscr{T}(E)$ is the complement of this basin.

We denote by $\mathscr{A}$ the set of points $z \in \mathbb{C} \backslash \bar{H}$ such that $E_{\lambda_{1}, \ldots, \lambda_{n}}(z) \in \mathbb{C} \backslash \bar{H}$. In the next stage, we examine the magnitude of the derivative $E_{\lambda_{1}, \ldots, \lambda_{n}}^{\prime}$ of $E_{\lambda_{1}, \ldots, \lambda_{n}}$ on $\mathscr{A}$. In fact we are going to prove that

$$
\left|E_{\lambda_{1}, \ldots, \lambda_{n}}^{\prime}(z)\right| \geq 1+\varepsilon
$$

for each $z \in \mathscr{A}$ by a contradiction. Suppose that $\left|E_{\lambda_{1}, \ldots, \lambda_{n}}^{\prime}(z)\right|<1+\varepsilon$ for some $z \in \mathscr{A}$. Let us write

$$
\left|E_{\lambda_{i}, \ldots, \lambda_{n}}(z)\right|=e^{A_{i}} \text { and } E_{\lambda_{i}, \ldots, \lambda_{n}}(p)=e^{P_{i}}
$$

for $i=1,2, \ldots, n$. Then

$$
\left|E_{\lambda_{i}, \ldots, \lambda_{n}}(z)\right| \geq E_{\lambda_{i}, \ldots, \lambda_{n}}(p)
$$

if and only if $A_{i} \geq P_{i}$ for each $i=1,2, \ldots, n$. For confirmation, we argue as follows:

We note the following two cases:

(1) If $j=1$, then since $z \in \mathscr{A}$, and thus $E_{\lambda_{1}, \ldots, \lambda_{n}}(z) \in \mathscr{A} \subset \mathbb{C} \backslash \bar{H}$,

$$
e^{A_{1}}=\left|E_{\lambda_{1}, \ldots, \lambda_{n}}(z)\right|>p=E_{\lambda_{1}, \ldots, \lambda_{n}}(p)=e^{p_{1}},
$$

and so $A_{1}>P_{1}$.

(2) If $j>1$, suppose that there exists $j$ such that $A_{j}<P_{j}$. We obtain the following consequence:

$$
e^{A_{j-1}}=e^{\lambda_{j-1} e^{A_{j}} \cos B_{j}} \leq e^{\lambda_{j-1} e^{A_{j}}}<e^{\lambda_{j-1} e^{P_{j}}}=e^{P_{j-1}}
$$

where $B_{j}$ is some real value. Hence $A_{j}<P_{j}$ implies $A_{j-1}<P_{j-1}$, and so in particular $A_{1}<P_{1}$ which contradicts case (1).

\footnotetext{
${ }^{1}$ Notice that $p \in \mathscr{T}(E)$, so $\mathscr{T}(E)$ is not strictly to the right of the line $x=p$. The idea of this argument is due to Devaney [6].
} 
Now we claim that (7) holds, for otherwise

$$
\begin{aligned}
\operatorname{Re} E_{\lambda_{1}, \ldots, \lambda_{n}}(z) & \leq\left|E_{\lambda_{1}, \ldots, \lambda_{n}}(z)\right|=\frac{\left|E_{\lambda_{1}, \ldots, \lambda_{n}}^{\prime}(z)\right|}{\prod_{i=1}^{n} \lambda_{i} \prod_{i=2}^{n}\left|E_{\lambda_{i}, \ldots, \lambda_{n}}(z)\right|} \\
& \leq \frac{\left|E_{\lambda_{1}, \ldots, \lambda_{n}}^{\prime}(z)\right|}{\prod_{i=1}^{n} \lambda_{i} \prod_{i=2}^{n} E_{\lambda_{i}, \ldots, \lambda_{n}}(p)} \\
& =\frac{p\left|E_{\lambda_{1}, \ldots, \lambda_{n}}^{\prime}(z)\right|}{E_{\lambda_{1}, \ldots, \lambda_{n}}^{\prime}(p)}<\frac{p(1+\varepsilon)}{1+\varepsilon}=p
\end{aligned}
$$

which contradicts $E_{\lambda_{1}, \ldots, \lambda_{n}}(z) \in \mathbb{C} \backslash \bar{H}$ and thus shows our assertion.

Let $D$ be a closed disc with radius $\delta$ and $E_{\lambda_{1}, \ldots, \lambda_{n}}^{k}(D) \subset \mathbb{C} \backslash \bar{H}$ for all $k \geq 0$. Then

$$
\left|E_{\lambda_{1}, \ldots, \lambda_{n}}^{\prime}\left(E_{\lambda_{1}, \ldots, \lambda_{n}}^{k}(z)\right)\right| \geq 1+\varepsilon
$$

for $z \in D$ and all $k$. Hence

$$
\left|\left(E_{\lambda_{1}, \ldots, \lambda_{n}}^{k}\right)^{\prime}(z)\right|=\prod_{i=1}^{k}\left|E_{\lambda_{1}, \ldots, \lambda_{n}}^{\prime}\left(E_{\lambda_{1}, \ldots, \lambda_{n}}^{i-1}(z)\right)\right| \geq(1+\varepsilon)^{k},
$$

which tends to $\infty$ as $k \rightarrow \infty$.

It follows from the Bloch-Landau Theorem that $E_{\lambda_{1}, \ldots, \lambda_{n}}^{k}(D)$ contains a disc with arbitrary large radius for $k$ sufficiently large. Thus there exists an integer $k_{0}>0$ such that $E_{\lambda_{1}, \ldots, \lambda_{n}}^{k_{0}}(D) \cap H \neq \varnothing$. But this is absurd. The contradiction shows the impossibility of $E_{\lambda_{1}, \ldots, \lambda_{n}}^{k}(D)$ staying in $\mathbb{C} \backslash \bar{H}$ for all $k$. Thus the complement of the basin of attraction of $q$ is nowhere dense in $\mathbb{C}$. As an immediate consequence, $\left\{E_{\lambda_{1}, \ldots, \lambda_{n}}^{k}\right\}_{k=0}^{\infty}$ is normal nowhere in the complement of the basin. This is equivalent to saying that $\mathscr{T}(E)$ is the complement of the basin of the attraction of $q$. Q.E.D.

Remark. In order to study the dynamical behavior of a given function $f$, we often need to investigate the possible limit functions of subsequences of $\left\{f^{n}\right\}_{n=0}^{\infty}$ in the set of normality. The ideas used in this work may also be applicable to more general functions in the following sense:

If we assume that $f$ is of critically finite type, the finiteness theorem combining Baker's results provides a useful tool for finding the relevant limit functions. In this case, the set $\mathscr{S}$ only consists of finitely many points. This implies that $\mathscr{E}$ is a countable set. If furthermore we assume the complement of $\mathscr{L}$ is connected and the interior of $\mathscr{L}$ is empty (this occurs, for instance, when $\mathscr{L}$ happens to be a countable set), then Baker's results enable us to give a better estimate of the possible limit functions. To precisely determine the limit functions of subsequences of $\left\{f^{n}\right\}_{n=0}^{\infty}$, we need to examine the order of the growth of $\left\{f^{n}\right\}_{n=0}^{\infty}$ in the domain concerned. One way to do this examination is to consider the sequence of derivatives $\left\{\left(f^{n_{k}}\right)^{\prime}\right\}_{k=0}^{\infty}$ of $\left\{f^{n_{k}}\right\}_{k=0}^{\infty}$. If some 
disc in the domain concerned is expanded under the iterations of $f$, with the Bloch-Landau Theorem we are able to establish a contradiction and conclude $\mathscr{T}(f)=\mathbb{C}$. To see an application to the family of the composition of sine functions with $n$ parameters, let

$$
S_{\lambda_{1}}(z)=\lambda_{1} \sin z
$$

and

$$
S_{\lambda_{1}, \ldots, \lambda_{k+1}}(z)=S_{\lambda_{1}, \ldots, \lambda_{k}}\left(\lambda_{k+1} \sin z\right)
$$

for $k=1, \ldots, n$. Since finite type maps are closed under composition by Lemma $2, S_{\lambda_{1}, \ldots, \lambda_{n}}$ is of critically finite type. It is easy to check that the finite singularities of $S_{\lambda_{1}, \ldots, \lambda_{n}}^{-1}$ are

$$
\pm \lambda_{1}, \lambda_{1} \sin \left( \pm \lambda_{2}\right), \ldots, S_{\lambda_{1}, \ldots, \lambda_{n-1}}\left( \pm \lambda_{n}\right) .
$$

With the same method as we used in the proof of Theorem 1, one can show that if each forward orbit of finite singularities of $S_{\lambda_{1}, \ldots, \lambda_{n}}^{-1}$ tends to $\infty$, then the Julia set of $S_{\lambda_{1}, \ldots, \lambda_{n}}$ is the whole plane.

\section{ACKNOWLEDGMENT}

The author is grateful to Professor Sanford Segal for valuable suggestions.

\section{REFERENCES}

1. I. N. Baker, Repulsive fixpoints of entire functions, Math. Z. 104 (1968), 252-256.

2. _ Limit functions and sets of nonnormality in iteration theory, Ann. Acad. Sci. Fenn. Ser. A I Math. 467 (1970), 1-10.

3. Soc. Ser. A 30 (1981), 483-495.

4. I. N. Baker and P. J. Rippon, Iteration of exponential functions, Ann. Acad. Sci. Fenn. Ser. A I Math. 9 (1984), 49-77.

5. R. Devaney, Julia sets and bifurcation diagrams for exponential maps, Bull. Amer. Math. Soc. 11 (1984), 167-171.

6. __ An introduction to chaotic dynamical systems, Benjamin/Cummings, 1986.

7. P. Fatou, Sur les equations fonctionelles, Bull. Soc. Math. France 47 (1919), 161-271.

8. __ Sur les equations fonctionelles, Bull. Soc. Math. France 48 (1920), 33-94, 208-313.

9. __ Sur l'itération des fonctions transcendantes entières, Acta Math. 47 (1926), 337-370.

10. L. Goldberg and L. Keen, A finiteness theorem for a dynamical class of entire functions, Ergodic Theory Dynamical Systems 6 (1986), 183-192.

11. M. Misiurewicz, On iterates of $e^{z}$, Ergodic Theory Dynamical Systems 1 (1981), 103-106.

12. D. L. Shell, On the convergence of infinite exponentials, Proc. Amer. Math. Soc. 13 (1962), 678-681.

13. W. J. Thron, Convergence of infinite exponentials with complex elements, Proc. Amer. Math. Soc. 8 (1957), 1040-1043.

Department of Mathematics, University of Wisconsin, Oshkosh, Wisconsin 54901 